\title{
PATIENTS' MEDICAL CHARTS IN INTENSIVE CARE UNIT: A POTENTIAL SOURCE OF NOSOCOMIAL INFECTIONS
}

\section{Roshan Thapa, Eliza Thapa, Anjana Singh, Bharat Mani Pokharel, Upendra Devkota}

Journal of Institute of Science and Technology Volume 21, Issue 1, August 2016

ISSN: 2469-9062 (print), 2467-9240(e)

Editors:

Prof. Dr. Kumar Sapkota

Prof. Dr. Armila Rajbhandari

Assoc. Prof. Dr. Gopi Chandra Kaphle

JIST, 21 (1), 48-51 (2016)

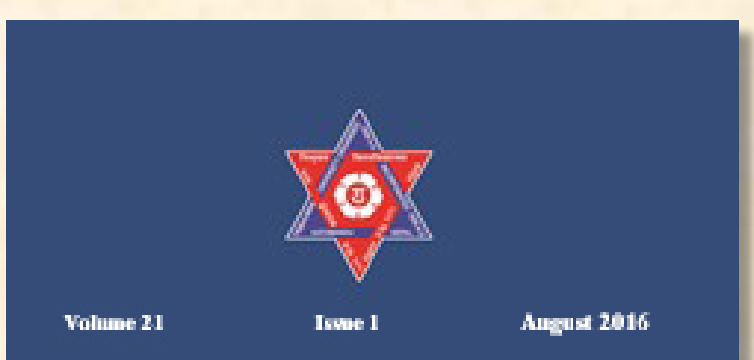

JOURNAL OF INSTITUTE OF SCIENCE AND TECHNOLOGY

Institute of Seience and Teehnology

Tribhuvan University

Kintipur, Kathmanda, Nepal

Published by:

Institute of Science and Technology

Tribhuvan University

Kirtipur, Kathmandu, Nepal 


\title{
PATIENTS' MEDICAL CHARTS IN INTENSIVE CARE UNIT: A POTENTIAL SOURCE OF NOSOCOMIAL INFECTIONS
}

\author{
Roshan Thapa $^{1, *}$, Eliza Thapa ${ }^{1}$, Anjana Singh ${ }^{1}$, Bharat Mani Pokharel $^{2}$, Upendra Devkota ${ }^{3}$ \\ ${ }_{2}^{1}$ Central Department of Microbiology, Tribhuvan University, Kirtipur, Nepal, \\ Institute of Medicine, Tribhuvan University, Maharagunj, Kathmandu, Nepal, \\ National Institute of Neurological and Allied Sciences, Bansbari, Kathmandu, Nepal \\ "Corresponding email: roshanthapa248@gmail.com
}

\begin{abstract}
This study was carried out to determine the degree of contamination of patients' medical charts in ICU settings. A total of 100 medical charts were randomly selected from ICU. The swab samples were collected from outer surface of charts with sterile swabs moistened with sterile normal saline. The swabs were immediately transferred to Trypticase soy broth and incubated aerobically for $48 \mathrm{~h}$ and then subcultured in MacConkey, Blood and Eosin-methylene blue agars. The isolates were identified by using standard microbiological and biochemical techniques. Out of 100 charts sampled, $89 \%$ charts were found to be contaminated with pathogenic or potentially pathogenic bacteria. Staphylococcus aureus was the most commonly isolated bacteria in ICU $(\mathrm{n}=32,28.3 \%)$ followed by Citrobacter freundii $(\mathrm{n}=27,24 \%)$, Coagulase Negative Staphylococci (CoNS) $(n=23,20.3 \%)$ and Acinetobacter spp. $(n=12,10.7 \%)$. It is concluded that medical chart contains pathogenic and potentially pathogenic bacteria. Thus, contaminated charts can act as vector of pathogenic bacteria and source of cross-infection in hospital.
\end{abstract}

Key words: Intensive care unit, Nosocomial infection, Patients' medical charts

\section{INTRODUCTION}

Hospital Acquired Infections (HAIs) or Nosocomial infections (NIs), significant cause of morbidity and mortality of hospitalized patients as well as a large financial burden worldwide, are becoming increasingly common worldwide (Marinella \& Elder 2000, Tolpadi et al. 2014). The highest prevalence of nosocomial infections occur in busy areas of hospital such as Intensive care units (ICUs) and wards in which patients undergo invasive procedures and frequent handling of infected wounds. Transmission route and source of infectious agent are two important elements in transmission of infection in hospital settings (Fridkin et al. 1997, Teng et al. 2009, Tolpadi et al. 2014). Susceptibility to such infections has been associated with use of invasive devices, extremes of age, immune status and infection control practices (Teng et al. 2009). Most nosocomial infections are thought to be transmitted by the hands of health care workers. It has long been known that hand hygiene among health care workers plays a vital role in preventing the transmission of infectious agents (Allegranzi et al. 2009, WHO, 2009). HAIs may be caused by infectious agents from endogenous (body sites) or exogenous sources (patient care personnel, visitors, patient care equipment, medical devices or the health care environment (Horan et al. 2008). Commonly used items such as stethoscopes, latex gloves, white coats, mobile phones, writing pens, case notes and medical charts have been noted to be contaminated with various bacterial species some of which are pathogenic (Loh et al. 2000, Cohen et al. 2003, Teng et al. 2009, Halton et al. 2011, Tekerekoglu et al. 2011, Chen et al, 2014). Patients' files are common inanimate objects, which have the potential to be contaminated by pathogenic bacteria, and work as fomites in spreading nosocomial infections.

Although a number of methods, including hand washing, have been used to minimize the occurrence of related infections, there has not been much focus on the source of potential infection in the environment acquired through inanimate objects, particularly, the role of hospital medical charts as a possible vector of pathogens (WHO 2009; Chen et al. 2014). Medical charts are 
routinely handled by multiple hospital personnel whose hands could potentially become contaminated with pathogenic bacteria after handling. The extent to which bedside files become contaminated and the range of bacterial flora attributable to contamination in high risk areas of hospital are not known with certainty. This crosssectional study was thus carried out with an aim to determine the degree of contamination of patients' medical charts in ICU settings and to find out prevalence of nosocomial microorganisms present on the surface of patients' medical charts.

\section{MATERIALS AND METHODS}

This cross-sectional study was conducted at National Institute of Neurological and Allied Sciences, Bansbari, Kathmandu, Nepal, from March 2014 to August 2014. A total of 100 medical chart samples were randomly collected from ICU $(n=100)$. The samples were collected from entire outer surface (plastic covers) of the selected medical charts with sterile cotton swabs moistened in normal saline by an investigator wearing sterile gloves. The sampled swabs were immediately inoculated into Trypticase soy broth and incubated for $48 \mathrm{~h}$ and then subculture in MacConkey, Blood and Eosin-methylene blue agars (Teng et al. 2009). The isolates from the cultures were identified using standard microbiological and biochemical techniques at hospital's microbiology laboratory. In case $S$. aureus was isolated from culture, then Methicillin Resistant Staphylococcus aureus (MRSA) was further confirmed by antibiotic susceptibility testing using Kirby-Bauer disk diffusion method.

\section{RESULTS AND DISCUSSION}

Of the 100 charts sampled in the ICU, 89 (89\%) were contaminated with pathogenic or potentially pathogenic bacteria of which $13(14.6 \%)$ showed monomicrobial and $76 \quad(85.4 \%)$ showed polymicrobial contamination. Similar results have been reported in the previous studies carried out by the different researchers (Teng et al. 2009, Chen et al. 2014). Eight out of 100 charts were contaminated with environmental flora. Only 3 charts were not contaminated by either pathogenic or environmental bacteria (Table 1). Research on contamination of patients' medical charts in a large district general hospital in the United Kingdom found a $99.6 \%$ contamination rate, but most of the isolates were environmental organisms, with $S$. aureus being isolated from $3.9 \%$ of charts and no gram-negative bacteria were isolated (Bebbington et al. 2003). Two studies in Saudi Arabia found that $57-100 \%$ of patients' charts were contaminated with pathogenic or potentially pathogenic bacteria (Alothman et al. 2003, Panhotra et al. 2005).

Table 1. Percentage of contamination of patients' medical charts in ICU

\begin{tabular}{|l|c|c|}
\hline \multicolumn{1}{|c|}{ Variable } & Total Number & Percentage (\%) \\
\hline Charts with pathogenic isolates & 89 & $\mathbf{8 9}$ \\
a) monomicrobial & 13 & 14.6 \\
b) polymicrobial & 76 & 85.4 \\
\hline Charts with environmental bacteria & 8 & 8 \\
\hline Charts without isolates & 3 & 3 \\
\hline Total & $\mathbf{1 0 0}$ & $\mathbf{1 0 0}$ \\
\hline
\end{tabular}

Table 2. Bacteria isolated from patients' medical charts in ICU

\begin{tabular}{|l|c|c|}
\hline \multicolumn{1}{|c|}{ Bacterial isolates } & Number & Percentage (\%) \\
\hline $\begin{array}{l}\text { A. Pathogenic } \\
\text { 1. Gram positive bacteria }\end{array}$ & & \\
\hline Coagulase-negative staphylococci & 23 & 20.3 \\
\hline S. aureus & 32 & $\mathbf{2 8 . 3}$ \\
\hline Enterococcus spp. & 7 & 6.2 \\
\hline Micrococcus spp. & 1 & 0.9 \\
\hline 2. Gram negative bacteria & 27 & 24 \\
\hline Citrobacter freundii & 4 & 3.5 \\
\hline C. koseri & 12 & 10.7 \\
\hline Acinetobacter spp. & & \\
\hline
\end{tabular}




\begin{tabular}{|l|c|c|}
\hline Pseudomonas aeruginosa & 2 & 1.7 \\
\hline Klebsiella pneumoniae & 3 & 2.7 \\
\hline K. oxytoca & 2 & 1.7 \\
\hline Total & $\mathbf{1 1 3}$ & $\mathbf{1 0 0}$ \\
\hline & & \\
\hline B. Environmental & & 91.6 \\
\hline Bacillus spp. & 76 & 8.4 \\
\hline Corynebacterium spp. & 7 & $\mathbf{1 0 0}$ \\
\hline Total & $\mathbf{8 3}$ & \\
\hline
\end{tabular}

In this study, 196 bacterial isolates were obtained from 97 culture positive medical charts, of which 113 were pathogenic or potentially pathogenic whereas 83 were environmental flora. The predominant organism was found to be environmental species (Bacillus spp.), which tend to occur in almost all medical chart $(\mathrm{n}=76,91.6 \%)$. Similar results were also reported by Marinella and Elder (2000). Among the pathogenic or potentially pathogenic isolates, $S$. aureus was the most predominating organism in ICU $(\mathrm{n}=32,28.3 \%)$. However, in other studies by Teng et al. (2009), Chen et al. (2014) and Tolpadi et al. (2014), it has been shown that Coagulase negative Staphylococci (CoNS) were the most predominating organism from ICU. Similarly, study carried out by Panhotra et al. (2005) reported Pseudomonas aeruginosa as the most predominating organism from ICU. After $S$. aureus, the most commonly isolated bacteria were Citrobacter freundii $(\mathrm{n}=27,24.0 \%)$, CoNS $(\mathrm{n}=23,20.3 \%)$ and Acinetobacter spp. $(\mathrm{n}=12$, $10.7 \%$ ) (Table 2). Similar results were reported in the study by Panhotra et al. (2005), Veena Kumari et al. (2012). However, other studies reported Streptococcus and Enterococcus spp. as the second most prevalent organism (Marinella \& Elder 2000, Teng et al. 2009, Chen et al. 2014). The prevalence of gram positive bacteria colonization was significantly higher than that of gram negative bacteria in ICU. This result is analogous to studies carried out by other researchers (Teng et al. 2009, Chen et al. 2014).

Among 32 isolates of $S$. aureus, methicillin resistance was found in 12 isolates $(37.5 \%)$. The occurrence of methicillin resistant $S$. aureus (MRSA) was slightly higher in this study compared to study carried out by other researchers (Panhotra et al. 2005, Hamza et al. 2007, Teng et al. 2009, Chen et al. 2014, Tolpadi et al. 2014). This difference might be due to different hospital settings, different patient natures and strict measures for formulation of definite antibiotics policy to reduce the incidence of MRSA followed by hospitals. Two isolates of enterococci showed resistance to Vancomycin. Zimbudzi et al. (2011) also demonstrated that the medical chart covers can be contaminated with Vancomycin-resistant enterococci (VRE).

\section{CONCLUSION}

Medical charts are handled by multiple hospital personnel while recording, looking-up and handing over to the next shifts. Also, medical charts are in continuous circulation throughout hospital, so they are prone to bacterial contamination. Based on the finding that most hospital charts are contaminated by bacteria, this study also confirms that a hospital chart is indeed not only a medical record but also an important source of potential infection. Occurrence of MRSA in chart covers also highlighted serious threat for patients in ICUs. Thus, patients' medical charts have the potential to act as vector of bacterial pathogens which once again highlighted the importance of effective hand washing before and after contact with patients' charts.

\section{ACKNOWLEDGEMENTS}

We would like to thank National Institute of Neurological And Allied Sciences (NINAS) for allowing us to conduct this study.

\section{REFERENCES}

Allegranzi, B. and Pittet, D. 2009. The role of hand hygiene in healthcare-associated infection prevention. Journal of Hospital Infection 73: 305-315.

Alothman, A., Jelani, A., Althaqafi, A., Rich, M. and Williams, E. 2003. Contamination of patient hospital charts by bacteria. Journal of Hospital Infection 55: 304-305.

Bebbington, A., Parkin, I., James, P. A., Chichester, L. J. and Kubiak, E. M. 2003. Patients' case- 
notes: look but don't touch. Journal of Hospital Infection 55: 299-301.

Chen, K. H., Chen, L. R. and Wang, Y. K. 2014. Contamination of medical charts: An important source of potential infection in hospitals. PLOS ONE 9(2): e78512.

Cohen, S. R., McCormack, D. J., Youkhana, A. and Wall, R. 2003. Bacterial colonization of stethoscopes and the effect of cleaning. Journal of Hospital Infection 55: 236-237.

Fridkin, S. K., Welbel, S.F. and Weinstein, R. A. 1997. Magnitude and prevention of nosocomial infections in the intensive care unit. Infectious Disease Clinics of North America 11: 479-496.

Halton, K., Arora, V., Singh, V., Ghantoji, S. S., Shah, D. N. and Garey, K. W. 2011. Bacterial colonization on writing pens touched by healthcare professionals and hospitalized patients with and without cleaning the pen with alcohol-based hand sanitizing agent. Clinical Microbiology and Infection 17: 868-869.

Hamza, N., Bazoua, G., Al-Shajerie, Y., Kubiak, E., James, P. and Wong, C. 2007. A prospective study of the case-notes of MRSA-positive patients: a vehicle of MRSA spread. Annals of the Royal College of Surgeons of England 89: 665-667.

Horan, T. C., Andrus, M. and Dudeck, M. A. 2008. CDC/NHSN surveillance definition of healthcare associated infection and criteria for specific type of infections in the acute care setting. American Journal of Infection Control 36(5): 309-332.

Loh, W., Ng, V. V. and Holton, J. 2000. Bacterial flora on the white coats of medical students. Journal of Hospital Infection 45: 65-68.

Marinella, M. A. and Elder, L. B. 2000. Bacterial contamination of patient hospital charts. Infectious Disease in Clinical Practice. 9: 39-40.
Panhotra, B. R., Saxena, A. K. and Al-Mulhim, A. S. 2005. Contamination of patients' files in intensive care units: an indication of strict handwashing after entering case notes. American Journal of Infection Control 33: 398-401.

Tekerekoglu, M. S., Duman, Y., Serinda, A., Cuglan, S. S., Kaysadu, H., Tunc, E. and Yakupogullari, Y. 2011. Do mobile phones of patients, companions and visitors carry multidrug-resistant hospital pathogens? American Journal of Infection Control 39(5): 379-381.

Teng, S. O., Lee, W. S., Ou, T. Y., Hsieh, Y. C., Lee, W. C. and Lin, Y. C. 2009. Bacterial contamination of patients' medical charts in a surgical ward and the intensive care unit: impact on nosocomial infections. Journal of Microbiology, Immunology and Infection 42(1): 86-91.

Tolpadi, A. G., Ravindra, J. S. and Sujeet, M. M. 2014. Contamination of patient's medical files in ICU: potential source of nosocomial infection. International Jounal of Health Sciences and Research 4(3): 223-225.

Veena Kumari, H. B., Nagarathna, S., Reddemma, K., Lalitha, K., Mary, B. and Sateesh, V.L. 2012. Containment of case-file contamination-infection control. Journal of Evolution of Medical and Dental Sciences 1(6): 1166-1171.

World Health Organization. 2009. WHO guidelines for hand hygiene in health care. Geneva, Switzerland: World Health Organization.

Zimbudzi, E., Stuart, R. L., Korman, T. M. and Kotsanas, D. 2011. Contamination of renal patients' hospital chart covers with vancomycin-resistant enterococci: Handle with care. Australasian Medical Journal 4: 538-541. 